Journal of Bangladesh Academy of Sciences, Vol. 34, No. 1, 49-58, 2010

\title{
AN ASYMPTOTIC INVESTIGATION OF THE NON-STATIONARY MODES OF INSTABILITY ON A ROTATING-DISK IN THE PRESENCE OF A LARGE MAGNETIC FIELD STRENGTH
}

\author{
H. A. JASMINE \\ Department of Mathematics, University of Rajshahi, Rajshahi, Bangladesh
}

\begin{abstract}
Asymptotic solutions for stationary and non-stationary modes for the upper and lower branch disturbances assuming large scale magnetic fields are investigated. A triple deck structure which governs the lower branch modes for a large scale magnetic field is displayed. The wavenumbers and waveangle calculated from the eigenrelations $\alpha \approx 2.62 \mathrm{~m}^{5 / 4} \mathrm{r}^{-1 / 2}, \beta \approx 0.78 \mathrm{~m}^{1 / 4} \mathrm{r}^{1 / 2}, \phi=0.298 \mathrm{~m}^{-}$

${ }^{1} r$, to be consistent with the numerical results for large scale magnetic fields.
\end{abstract}

Key words: Magnetic field, Instability, Rotating-disk flow

\section{INTRODUCTION}

Studies have been carried out to analyze the phenomenon of instability of a three dimensional boundary layer flow of an incompressible fluid on an infinite rotating disk. It is the need to comprehend the instability of the boundary layer on a swept wing that has inspired most of these investigations. The design of laminar flow control has promise of significant improvement in airplane fuel efficiency. Malik (1986) in a parallel flow approximation computed the value of $\phi=11^{\circ}$ corresponding to the critical Reynolds number. Hall (1986) discussed the high Reynolds number asymptotic structure of the viscous mode of Malik (1986) and pointed out that this lower branch mode was controlled by a triple-deck structure fixed by a balance between viscous and Coriolis forces. Following the procedure developed by Hall (1986), and taking into account nonlinear effects, Mackerrell (1987) extended Hall's asymptotic structure to derive an amplitude equation governing the growth of disturbances near the neutral location. It was shown that with nonlinearity, the instability was subcritical.

Hall (1986) has given asymptotic analysis of the upper branch stationary modes, i.e. zero-frequency waves. Turkyilmazoglu and Gajjar (1998) (hereafter referred to as TG) extended Hall's work to consider non-zero frequency waves for the upper branch instability modes. An eigenrelation involving the correction terms for the wavenumber and waveangle was derived. The wavenumbers for different frequencies so computed agree well with the numerical results. In this paper, we have extended investigations by Hall (1986) and TG for zero frequency and for non-zero frequency, respectively, by imposing on the flow a magnetic field normal to the rotating disk. Jasmine and Gajjar (2005) have given numerical solution of the absolute and convective instability in the 
incompressible boundary layer on a rotating disk in the presence of a uniform magnetic field. We have derived asymptotic solutions assuming large magnetic fields.

This paper is organized as follows. The equations governing the flow of an infinite disk rotating in an incompressible fluid with an imposed external magnetic field normal to the disk are formulated in section 2. An asymptotic structure of the upper branch mode when the magnetic field is large is developed in Section 3. A triple-deck structure which governs the lower branch modes for large scale magnetic fields is given in Section 4. The conclusions are set in Section 5.

Mathematical formulation

Consider the three dimensional boundary layer flow of an incompressible, electrically conducting viscous fluid on an infinite disk which rotates about its axis with a constant angular velocity $\Omega$. A uniform magnetic field $\mathbf{B}=B_{0} \mathbf{k}$ is applied to the system, where $\mathbf{k}$ is a unit vector parallel to the $\mathrm{z}$-axis. If the magnetic Reynolds number is small $\left(R_{m}<<1\right)$, then within the viscous boundary layer, the electric and magnetic fields, $\mathbf{E}$ and $\mathbf{B}$, will not be significantly different from their values at the disk surface. This is linked to the idea that the viscous boundary layer is usually much thinner than the magnetic boundary layer. Then, the current density $\mathbf{J}=\left(\sigma B_{0} v, \sigma B_{0} u, 0\right)$. In order to nondimensionalize the Navier-Stokes equations, we introduce non-dimensional quantities $r^{*}$, $\theta^{*}, z^{*}, t^{*}, u^{*}, v^{*}, w^{*}, \mathbf{B}^{*}$, and $P^{*}: r=L r^{*}, z=L z^{*}, \theta=\theta^{*}, t=t^{*}{ }^{*} U_{c}, P=\rho U^{2}{ }_{\mathrm{c}} P^{*}, u=U_{c} u^{*}, v$ $=U_{c} v^{*}, w=U_{c} w^{*}, \mathbf{B}^{*}=B_{0} \mathbf{B}$, where $\mathrm{L}$ is a given length scale and $U_{c}=L \Omega$ is a given velocity scale. For convenience of writing, we shall suppress the * over the nondimensional variables. The full time-dependent, unsteady Navier-Stokes equations governing the viscous fluid flow are the usual momentum and the continuity equations given as follows:

$$
\begin{aligned}
& \frac{\partial u}{\partial t}+u \frac{\partial u}{\partial r}+\frac{v}{r} \frac{\partial u}{\partial \theta}+w \frac{\partial u}{\partial z}-\frac{v^{2}}{r}-2 v-r \\
& =-\frac{\partial P}{\partial r}+\frac{1}{R^{2}}\left[\nabla^{2} u-\frac{2}{r^{2}} \frac{\partial v}{\partial \theta}-\frac{u}{r^{2}}\right]-\frac{\sigma B_{0}^{2}}{\rho \Omega} u \\
& \frac{\partial v}{\partial t}+u \frac{\partial v}{\partial r}+\frac{v}{r} \frac{\partial v}{\partial \theta}+w \frac{\partial v}{\partial z}+\frac{u v}{r}+2 u \\
& =-\frac{1}{r} \frac{\partial P}{\partial \theta}+\frac{1}{R^{2}}\left[\nabla^{2} v+\frac{2}{r^{2}} \frac{\partial u}{\partial \theta}-\frac{v}{r^{2}}\right]-\frac{\sigma B_{0}^{2}}{\rho \Omega}(v+1), \\
& \frac{\partial w}{\partial t}+u \frac{\partial w}{\partial r}+\frac{v}{r} \frac{\partial w}{\partial \theta}+w \frac{\partial w}{\partial z}=-\frac{\partial P}{\partial z}+\frac{1}{R^{2}}\left[\nabla^{2} w\right],
\end{aligned}
$$




$$
\frac{\partial u}{\partial r}+\frac{1}{r} \frac{\partial v}{\partial \theta}+\frac{\partial w}{\partial z}+\frac{u}{r}=0
$$

where the velocity field with radial, tangential and vertical components are as $u, v$, and $w$, respectively. $P$ is the pressure. The quantities $\rho, v, \mu$, and $\sigma$ which represent mass density, kinematic viscosity, magnetic permeability, and electrical conductivity of the fluid, respectively, are all assumed to be constant throughout the flow field. Here, we have a global Reynolds number $R_{e}=U_{c} L / v=R^{2}$, where $R$ is the Reynolds number based on the displacement thickness $\delta=(v / \Omega)^{1 / 2}$. The Laplacian operator in cylindrical coordinates is

$$
\nabla^{2}=\left(\frac{\partial^{2}}{\partial r^{2}}+\frac{1}{r^{2}} \frac{\partial^{2}}{\partial \theta^{2}}+\frac{\partial^{2}}{\partial z^{2}}+\frac{1}{r} \frac{\partial}{\partial r}\right)
$$

Throughout this analysis, the fluid is assumed to lie in the semi-infinite space $z \geq 0$.

The basic velocity field $\left(u_{B}, v_{B}, w_{B}, p_{B}\right)=\left[r F(Z), r G(Z), 1 / R H(Z), 1 / R^{2} P(Z)\right]$. The basic magnetic field $\left(B_{r b}, B_{\theta b}, B_{z b}\right)=\left[0,0, B_{0}\right]$. The boundary layer coordinate $Z$, which is of order $O(1)$, is defined as $Z=z R$.

The self-similar equations take the form

$$
\begin{aligned}
& F^{2}-(G+1)^{2}+F^{\prime} H-F^{\prime \prime}+m F=0, \\
& 2 F(G+1)+G^{\prime} H-G^{\prime \prime}+m(G+1)=0, \\
& P^{\prime}+H^{\prime} H-H^{\prime \prime}=0, \\
& 2 F+H^{\prime}=0,
\end{aligned}
$$

where $m=\sigma B^{2}{ }_{0} / \rho \Omega$ is the magnetic interaction parameter assumed to be a constant. Here, primes denote derivatives with respect to $Z$. The appropriate boundary conditions are $F=G=H=0$ at $Z=0, F=0, G=-1, H=h_{\propto}$ as $Z \rightarrow \infty$

The value of $h_{\propto}$ is a constant vertical velocity of the rotating fluid in the far-field above the disk. For large $\mathrm{m}$, the equations (5 - 8) can be solved analytically. We expand the basic velocity components as

$$
F=m^{-1} F_{0}(\bar{z})+\cdots, G=G_{0}(\bar{z})+\cdots, H=m^{-3 / 2} H_{0}(\bar{z})+\cdots,
$$

where $\bar{Z}=m^{1 / 2} Z$. Substituing the above expansions into the equations (5 - 8) and equating terms of order one, we obtain

$$
\left(G_{0}+1\right)-G_{0}^{\prime \prime}=0, F_{0}-\left(G_{0}+1\right)^{2}-F_{0}^{\prime \prime}=0,2 F_{0}+H_{0}^{\prime}=0 .
$$

The solution of this system is

$$
F_{0}=1 / 3\left(e^{-\bar{z}}-e^{-2 \bar{z}}\right), G_{0}=\left(e^{-\bar{z}}-1\right), H_{0}=2 / 3\left(e^{-\bar{z}}+1 / 2 e^{-2 \bar{z}}\right)-1 / 3
$$


Substituting for $F_{0}, G_{0}$, and $H_{0}$ into equation (10) yields

$$
\begin{aligned}
& F=m^{-1}\left[\frac{1}{3}\left(e^{-\bar{z}}-e^{-2 \bar{z}}\right)\right]+\cdots, \\
& G=\left(e^{-\bar{z}}-1\right)+\cdots, \\
& H=m^{-\frac{2}{3}}\left[\frac{2}{3}\left(e^{-\bar{z}}-e^{-2 \bar{z}}\right)-\frac{1}{3}\right]+\cdots,
\end{aligned}
$$

We must solve equations ( 1 - 4) subject to the no-slip condition at the wall, whereas sufficiently far away from the wall we insist that the disturbances decays to zero. We are going to consider the lower-branch disturbances, which have a triple-deck structure for $R$ $>1$.

The Inviscid Zone

Following Hall (1986), we consider zero frequency inviscid modes. We assume that the disturbances for the upper branch take the form

$$
[U, V, W, P]=[u(z), v(\mathrm{z}), w(\mathrm{z}), p(\mathrm{z})] e^{\left(\frac{i}{\varepsilon^{3}}\right)\left\{\int \alpha(r, \varepsilon) d r+\theta \beta(\varepsilon)\right\}} e^{-\frac{i}{\varepsilon^{2}} c t}
$$

where $\varepsilon=R^{-1 / 6}$. The scaling chosen for the frequency is consistent with the asymptotic analysis of Bassom and Gajjar (1988). We shall also consider only neutral disturbances at the position $r$. On the upper branch, we expand the wavenumbers and frequency as $(\alpha, \beta$, $c)=\left(\alpha_{0}, \beta_{0}, c_{0}\right)+\varepsilon\left(\alpha_{1}, \beta_{1}, c_{1}\right)+\ldots$, assuming the existence of an inviscid zone depth $O\left(\varepsilon^{-}\right.$ ${ }^{3}$ ) as elucidated by Hall (1986). To satisfy the no-slip condition on the velocity at the disk wall, a viscous layer must exist.

In this region we expand $u, v, w$, and $p$ in the form $u=u_{0}(\varsigma)+\varepsilon u_{1}(\varsigma)+\ldots, v=v_{0}(\varsigma)$ $+\varepsilon v_{1}(\varsigma)+\ldots, w=w_{0}(\varsigma)+\varepsilon w_{1}(\varsigma)+\ldots, p=p_{0}(\varsigma)+\varepsilon p_{1}(\varsigma)+\ldots$, where $\varsigma=m^{1 / 2} \varepsilon^{-3} z$. Here $m=\sigma B^{2}{ }_{0} / \rho \Omega$ denotes the magnetic field parameter which is initially taken to be of $O(1)$, so that the above expansions in power of $\varepsilon$ hold. Later we consider the limit m large for zeroth order problem. The above expansions are then substituted into linearized N-S

equations (1 - 4) with $\frac{\partial}{\partial r}$ replaced by $\frac{\partial}{\partial r}+\left(i / \varepsilon^{3)}\left\{\alpha_{0}+\varepsilon \alpha_{1}+\ldots\right\}\right.$ and with $\frac{\partial}{\partial \theta}$ replaced by $\left(i / \varepsilon^{3)}\left\{\beta_{0}+\varepsilon \beta_{1}+\ldots\right\}\right.$. Equating terms of order $\varepsilon^{-3}$, we obtain

$$
\begin{aligned}
& i U_{B} u_{0}+m^{1 / 2} r w_{0} F^{\prime}=-i \alpha_{0} p_{0}, \quad i U_{B} \mathrm{v}_{0}+m^{1 / 2} r \quad w_{0} G^{\prime}=-\frac{i \beta_{0}}{r} p_{0} \\
& i U_{B} w_{0}=-m^{1 / 2} P_{0}^{\prime}, i \alpha_{0} u_{0}+\left(i \beta_{0} / r\right) \nu_{0}+m^{1 / 2} w_{0}^{\prime}=0, \\
& U_{B}=\alpha_{0} r F+\beta_{0} G .
\end{aligned}
$$

If $u_{0}, v_{0}$ and the pressure $\mathrm{p}_{0}$ from the above equations are eliminated, we find that $w_{0}$ satisfies $U_{B}\left(G_{0}^{\prime \prime}-\bar{\gamma}^{2} w_{0}\right)-U_{B}^{\prime \prime} w_{0}=0$. 
Here, $\bar{\gamma}^{2}=m^{-1} \gamma_{0}^{2}$, where $\gamma_{0}^{2}=\alpha_{0}^{2}+\beta_{0}^{2} / r^{2}$ is the effective wavenumber and $U_{B}=$ $\alpha_{0} r F+\beta_{0} G$ is the effective velocity profile. Primes denote derivative with respect to $\varsigma$. The boundary conditions are $w_{0}=0$ at $\varsigma=0, \infty$. For large $m, F=m^{-1}\left[1_{3}^{1}\left(e^{-\varsigma}-e^{-2 \varsigma}\right)\right]+\ldots$ and $G=\left(e^{-\varsigma}-1\right)+\ldots$ Equation (18) yields $U_{B} \approx \alpha_{0} r m^{-1} F_{0}+\beta_{0} G_{0}, U_{B}^{\prime \prime} \approx \alpha_{0} r m^{-1} F_{0}^{\prime \prime}+\beta_{0}$ $G_{0}^{\prime \prime}$, where $\left.F_{0}=1 /{ }_{3}\left(e^{-\varsigma}-e^{-2 \varsigma}\right)\right]$ and $G_{0}=\left(e^{-\varsigma}-1\right)$. For stationary modes of Rayleigh equation (19), the solution is restricted such that $U_{B}$ and $U_{B}^{\prime \prime}$ vanish at the same non-zero value of $\varsigma=\bar{\varsigma}$. This eigenvalue problem for the non-magnetic case was solved by Hall [2]. For the magnetic case, from the condition $U_{B}=0$ and $U^{\prime \prime}{ }_{B}=0$ at $\varsigma=\bar{\varsigma}$, we obtain, for large $m$

$$
\frac{\beta_{0}}{r \alpha_{0}}=\frac{1}{9 m}, \alpha_{0} \approx \overline{\gamma m}^{\frac{1}{2}}
$$

Here, $\bar{\gamma}$ is $O(1)$ constant, as follows from the solution of (19). The predictions (20) are tested against the full numerical solution given by Jasmine and Gajjar [5] for large $m$ at a fixed Reynolds number. Fig. 1b shows that the results are consistent with those in equation (20).

The wall modes

Hall (1986) has shown that lower branch disturbances having a triple-deck structure of the type discussed by Smith (1979) for Blasius flow can also exist. In this section, we will extend his formulation in the presence of a large magnetic field strength $M$. Firstly, we define the appropriate triple-deck structure based on the small parameter $\varepsilon=R^{1 / 16}$ where the thicknesses of the lower, main, and upper deck are $\varepsilon^{9}, \varepsilon^{8}$, and $\varepsilon^{4}$, repectively. The disturbances structure in the main and upper decks is essentially the same for the lower branch as determined by Smith (1979). On the lower branch, we assume that the disturbance structure takes the form

$$
[U, V, W, P]=[U(\mathrm{z}), V(\mathrm{z}), W(\mathrm{z}), P(\mathrm{z})] e^{\left.\left(\frac{i}{\varepsilon^{4}}\right) \iint \alpha(r, \varepsilon) d r+\theta \beta(\varepsilon)\right\}}
$$

On the lower branch, we also expand the wavenumbers $(\alpha, \beta)=\left(\alpha_{0}, \beta_{0}\right)+\varepsilon^{2}\left(\alpha_{1}, \beta_{1}\right)+\ldots$ Here, we have anticipated that the order $\varepsilon$ terms are zero, and we again seek $\alpha_{i}, \beta_{i}(i=0$, $1,2, \ldots)$, such that the flow is neutrally stable at the location $\mathrm{r}$. We define $\xi$, $\zeta$ and $Z$ by $\xi=z \varepsilon^{-9}, \zeta=z \varepsilon^{-8}, Z=z \varepsilon^{-4}$.

The upper deck

In the upper deck $F=0, G=-1$ and $Z=z \varepsilon^{-4}$. The perturbation quantities then take the form $(U, V, W, P)=\varepsilon^{3}\left(U_{0}(Z), V_{0}(Z), W_{0}(Z), P_{0}(Z)\right)+\varepsilon^{4}\left(U_{1}(Z), V_{1}(Z), W_{1}(Z), P_{1}(Z)\right)+$ ... . Substitution in the Navier-Stokes equations $(1-4)$, the zero-order equations imply

$$
\beta_{0} U_{0}=\alpha_{0} P_{0}, \beta_{0} V_{0}=\beta_{0} P_{0} / r, i \beta_{0} W_{0}=\frac{d P_{0}}{d \bar{z}}, \quad i \alpha_{0} U_{0}+i \beta_{0} V_{0} / r+\frac{d W_{0}}{d \bar{z}}=0
$$


When $z \rightarrow \propto$, the solution of this system that decays to zero is

$$
U_{0}=\left(\alpha_{0} / \beta_{0}\right) C e^{-\gamma z}, V_{0}=(C / r) e^{-\gamma_{0} z}, \quad W_{0}=\left(i \gamma_{0} / \beta_{0}\right) C e^{-\gamma_{0} z}, P_{0}=C e^{-\gamma_{\circ} z},
$$

where $\gamma_{0}^{2}=\left(\alpha_{0}^{2}+\beta_{0}^{2} / r^{2}\right)$ and $C$ is an unknown function of $r$.

The main deck

In the main deck, we define $\zeta=z \varepsilon^{-4}$. The disturbance then expands as $U=(1 / \varepsilon) u_{0}(\zeta)$ $+u_{1}(\zeta)+\ldots, V=(1 / \varepsilon) v_{0}(\zeta)+v_{1}(\zeta)+\ldots, W=\varepsilon^{3} w_{0}(\zeta)+\varepsilon^{4} w_{1}(\zeta)+\ldots, P=\varepsilon^{3} C+\varepsilon^{4} P_{1}(\zeta)+$ $\ldots$, where we have anticipated that $P$ is independent of $\zeta$ to the order $\varepsilon^{3}$ and therefore equal to $C$. Substituting into equations $(1-4)$, we find that $u_{0}, v_{0}, w_{0}$ satisfy $i \alpha_{0} r F u_{0}+$ $i \beta_{0} G u_{0}+r F^{\prime} w_{0}=0$,

$$
\begin{aligned}
& i \alpha_{0} r F v_{0}+i \beta_{0} G v_{0}+r G^{\prime} w_{0}=0, \\
& i \alpha_{0} u_{0}+\left(\frac{i \beta_{0}}{r}\right) v_{0}+\frac{d w_{0}}{d \varsigma}=0,
\end{aligned}
$$

where $F=m^{-1}\left[1 / 3\left(e^{-\zeta}-e^{-2 \zeta}\right)\right]+\ldots$ and $G=\left(e^{-\zeta}-1\right)+\ldots$ are mean velocity profiles. Primes denote derivative with respect to $\zeta$. Solution of this system that matches with the upper deck solution is

$$
u_{0}=\operatorname{Cr} \gamma_{0} F^{\prime} / \beta_{0}^{2}, v_{0}=\operatorname{Cr} \gamma_{0} G^{\prime} / \beta_{0}^{2}, w_{0}=-\left(i C \gamma_{0} / \beta_{0}\right)\left(\alpha_{0} r F+\beta_{0} G\right),
$$

where $\mathrm{F}$ and $\mathrm{G}$ are mean velocity profiles. We note from (23) that $\mathrm{w}_{0}$ in fact satisfies the no-slip condition when $\zeta \rightarrow 0$; however, unless $F^{\prime}$ and $G^{\prime}$ both vanish at $\zeta=0$, the other velocity components are non-zero there. If we choose $\alpha_{0}$ and $\beta_{0}$ such that

$$
\left(\alpha_{0} F^{\prime}(0)+\frac{\beta_{0}}{r} G^{\prime}(0)\right)=0,
$$

which gives for large $\mathrm{m}, \frac{\beta_{0}}{r \alpha_{0}}=\frac{1}{3 m}$, then $\left(\alpha_{0} u_{0}+\frac{\beta_{0} v_{0}}{r}\right) \rightarrow 0$ when $\zeta \rightarrow 0$.

The lower deck

In the lower deck, we expand $F$, $G$ for small $\zeta$ and write $\xi=\zeta / \varepsilon$, we have

$$
F=\varepsilon F_{0} \xi+\varepsilon^{2} F_{1} \xi^{2}+\varepsilon^{3} F_{2} \xi^{3}+\ldots, G=\varepsilon G_{0} \xi+\varepsilon^{2} G_{1} \xi^{2}+\varepsilon^{3} G_{2} \xi^{3}+\ldots \ldots,
$$

where $F_{j-1}=F^{j} / j$ !, $G_{j-1}=G^{j} / j$ ! ; for $j=1,2, \ldots$ To match with the solution (23), written in terms of $\xi$, and using (25), we expand therefore the lower-deck disturbances in the form

$$
\begin{aligned}
& U=\frac{r \gamma_{0} C}{\varepsilon \beta_{0}^{2}}\left[F_{0}+2 \varepsilon F_{1} \xi+\ldots\right]+\frac{U_{-1}(\xi)}{\varepsilon}+U_{0}(\xi)+\varepsilon U_{1}+\ldots, \\
& V=\frac{r \gamma_{0} C}{\varepsilon \beta_{0}^{2}}\left[G_{0}+2 \varepsilon G_{1} \xi+\ldots\right]+\frac{V_{-1}(\xi)}{\varepsilon}+V_{0}(\xi)+\varepsilon V_{1}(\xi) \ldots,
\end{aligned}
$$




$$
\begin{aligned}
& W=-\frac{r \gamma_{0} \varepsilon^{5} C}{\beta_{0}^{2}}\left[\left(\alpha_{0} F_{1} r+\beta_{0} G_{1}\right) \xi^{2}+\ldots\right]+\varepsilon^{6} W_{1} \xi+\ldots \\
& P=\varepsilon^{3} P_{1}(\xi)+\ldots
\end{aligned}
$$

We must now substitute the above expansions into the disturbance equations and solve for $\left(U_{-1}, V_{-1}\right),\left(U_{0}, V_{0}\right),\left(U_{1}, V_{1}, W_{1}, P_{1}\right)$ etc. From the continuity equation, we obtain immediately that

$$
V_{-1}=-\left(\frac{\alpha_{0} r}{\beta_{0}}\right) U_{-1}, V_{0}=-\left(\frac{\alpha_{0} r}{\beta_{0}}\right) U_{0} .
$$

The radial momentum equation then yields

$$
\begin{aligned}
& -i\left[r \alpha_{0} F_{1}+\beta_{0} G_{1}\right] \xi^{2} U_{1}+\frac{d^{2} U_{-1}}{d \xi^{2}}=0, \\
& -i\left[r \alpha_{0} F_{1}+\beta_{0} G_{1}\right] \xi^{2} U_{0}+\frac{d^{2} U_{0}}{d \xi^{2}}=-r F_{0} W_{1}+\left[r \alpha_{0} F_{2}+\beta_{0} G_{2}\right] \xi^{3} U_{-1}
\end{aligned}
$$

which must be solved subject to $U_{-1}=-r \gamma_{0} C F_{0} / \beta_{0}^{2}, U_{0}=0, \xi=0, U_{-1}, U_{0} \rightarrow 0, \xi$ $\rightarrow \infty$. The function $U_{-1}$ is given by $U_{-1}=-\frac{r \gamma_{0} C F_{0}}{\beta_{0}^{2}} \frac{U\left(0,2^{1 / 2} \Delta^{1 / 2} \xi\right)}{U(0,0)}$, where $\Delta=i\left(r \alpha_{0} F_{1}+\beta_{0} G_{1}\right)$ and $U\left(0,2^{1 / 2} \Delta^{1 / 4} \xi\right)$ is a parabolic cylinder function. The next order approximation to the radial and azimuthal momentum equations gives

$$
\begin{aligned}
& i\left(r \alpha_{0} F_{1}+\beta_{0} G_{1}\right) \xi^{2} U_{1}+2 r \xi F_{1} W_{1}+\frac{r \gamma_{0} C}{\beta_{0}^{2}}\left[r \alpha_{1} F_{0}+\beta_{1} G_{0}\right] 2 \xi^{2} F_{1}-2 V_{-1} \\
& =-\alpha_{0} P_{1}+\frac{d^{2} U}{d \xi^{2}}-m U_{-1} \\
& i\left(r \alpha_{0} F_{1}+\beta_{0} G_{1}\right) \xi^{2} V_{1}+2 r \xi G_{1} W_{1}+\frac{r \gamma_{0} C}{\beta_{0}^{2}}\left[r \alpha_{1} F_{0}+\beta_{1} G_{0}\right] 2 \xi^{2} G_{1}+2 U_{-1} \\
& =-\frac{\beta_{0}}{r} P_{1}+\frac{d^{2} U}{d \xi^{2}}-m V_{-1} .
\end{aligned}
$$

If we multiply these equations by $i \alpha_{0}$ and $i \beta_{0} / r$, respectively and add them, we obtain

$$
\begin{aligned}
& i\left(\alpha_{0} \frac{d^{2} U_{1}}{d \xi^{2}}+\frac{\beta_{0}}{r} \frac{d^{2} V_{1}}{d \xi^{2}}\right)+\gamma_{0}^{2} P_{1}+2 i \alpha_{0} V_{-1}-\frac{2 i \beta_{0} U_{-1}}{r}= \\
& i M\left(r \alpha_{0} F_{1}+\beta_{0} G_{1}\right)\left(i \alpha_{0} U_{1}+\frac{i \beta_{0} V_{1}}{r}\right) \xi^{2}+2 i \xi\left(r \alpha_{0} F_{1}+\beta_{0} G_{1}\right) W_{1} \\
& -2 i\left(r \alpha_{1} F_{0}+\beta_{1} G_{0}\right) \frac{\gamma_{0} C}{\beta_{0}^{2}}\left(r \alpha_{0} F_{1}+\beta_{0} G_{1}\right) \xi^{2} .
\end{aligned}
$$


The z-momentum and continuity equations furnish

$$
\begin{aligned}
& \frac{d P_{1}}{d \xi}=0, \rightarrow \quad P_{1}=C, \\
& i\left(\alpha_{0} U_{1}+\frac{\beta_{0}}{r} V_{1}\right)+\frac{d W_{1}}{d \xi}=\frac{i \gamma_{0} C}{\beta_{0}^{2}}\left(r \alpha_{1} F_{0}+\beta_{1} G_{0}\right)-i \alpha_{1} U_{-1}-\frac{i \beta_{1} V_{-1}}{r}
\end{aligned}
$$

We can eliminate $U_{1}, V_{1}$ from equations (31), (32) and (33) to get

$$
\frac{d^{3} W_{1}}{d \xi^{3}}-i A \xi^{2} \frac{d W_{1}}{d \xi}+2 i A \xi W_{1}=\gamma_{0}^{2} C+A B \xi^{2}+\frac{2 i \gamma_{0} C}{\beta_{0}}\left(1+\frac{G_{0}^{2}}{F_{0}^{2}}\right) F_{0} \frac{U(0, \sqrt{2 S})}{U(0,0)},
$$

where $A=\left(r \alpha_{0} F_{1}+\beta_{0} G_{1}\right), B=\frac{\gamma_{0} C}{\beta_{0}^{2}}\left(r \alpha_{1} F_{0}+\beta_{1} G_{0}\right), S=\Delta^{1 / 2} \xi$. We write the solution of equation (34) in the form

$$
\begin{aligned}
W_{1}= & -i\left(r \alpha_{1} F_{0}+\beta_{1} G_{0}\right) \frac{\gamma_{0} C \xi}{\beta_{0}^{2}}+ \\
& \Delta^{-\frac{3}{4}}\left[\gamma_{0}^{2} C Q_{1}(s)+\frac{2 i \gamma_{0} C}{\beta_{0}}\left(1+\frac{G_{0}^{2}}{F_{0}^{2}}\right) \frac{F_{0} Q_{2}(s)}{U(0,0)}\right]+K \xi^{2},
\end{aligned}
$$

where $K$ is a constant and $Q_{1}$ and $Q_{2}$ satisfy $Q^{\prime \prime}{ }_{1}-S^{2} Q^{\prime}{ }_{1}+2 S Q_{1}=1, Q_{1}(0)=Q_{1}(\propto)=0$, $Q^{\prime \prime}{ }_{2}-S^{2} Q_{2}^{\prime}+2 S Q_{2}=U\left(0,2^{1 / 2} S\right), Q_{2}(0)=Q_{2}(\propto)=0$. In fact, it is possible to express $Q_{1}, Q_{2}$ in terms of integrals involving parabolic cylinder functions. It remains for us to satisfy $U_{1}=V_{1}=0$ at $\xi=0$. With equations (32), (33) and (35) as in Hall (1986), this condition leads to the eigenrelation:

$$
\gamma_{0}^{2} I_{3}+\frac{i \gamma_{0} F_{0}}{\beta_{0}}\left(1+\frac{G_{0}^{2}}{F_{0}^{2}}\right) I_{4}=i \Delta^{\frac{1}{2}}\left(r \alpha_{1} F_{0}+\beta_{1} G_{0}\right) \frac{\gamma_{0}}{\beta_{0}^{2}} .
$$

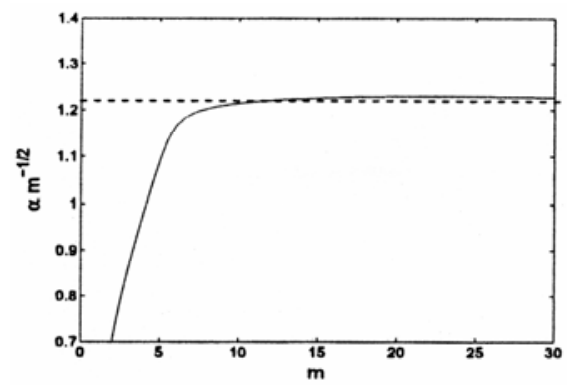

(a)

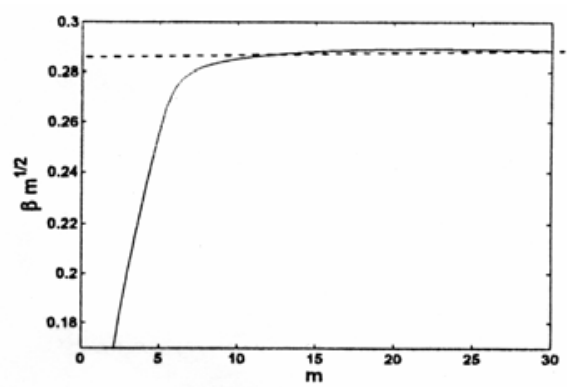

(b)

Fig. 1. Magnetic field $\mathrm{m}$ versus (a) $\alpha \mathrm{m}^{-1 / 2}$ and (b) $\beta m^{1 / 2}$ for fixed Reynolds number $R=1000$. The long curves show numerical results and doted lines show asymptotic ones. 
To separate real and imaginary parts, we express equation (36) in the form

$$
\begin{gathered}
\gamma_{0}^{2} I_{3}+\frac{i \gamma_{0} F_{0}}{\beta_{0}}\left(1+\frac{G_{0}^{2}}{F_{0}^{2}}\right) I_{4}=\frac{\gamma_{0} C F_{0}}{\beta_{0}^{2} 2^{1 / 2}}\left(\alpha_{0} r\right)^{1 / 2}\left(\alpha_{1}-\frac{\alpha_{0} \beta_{1}}{\beta_{0}}\right) \\
+i \frac{\gamma_{0} C F_{0}}{\beta_{0}^{2} 2^{1 / 2}}\left(\alpha_{0} r\right)^{1 / 2}\left(\alpha_{1}-\frac{\alpha_{0} \beta_{1}}{\beta_{0}}\right)
\end{gathered}
$$

Equating real and imaginary parts, we obtain

$$
\begin{aligned}
& \gamma_{0}^{2} I_{3}=\frac{\gamma_{0} C F_{0}}{\beta_{0}^{2} 2^{1 / 2}}\left(\alpha_{0} r\right)^{1 / 2}\left(\alpha_{1}-\frac{\alpha_{0} \beta_{1}}{\beta_{0}}\right) \\
& \frac{\gamma_{0} F_{0}}{\beta_{0}}\left(1+\frac{G_{0}^{2}}{F_{0}^{2}}\right) I_{4}=\frac{\gamma_{0} C F_{0}}{\beta_{0}^{2} 2^{1 / 2}}\left(\alpha_{0} r\right)^{1 / 2}\left(\alpha_{1}-\frac{\alpha_{0} \beta_{1}}{\beta_{0}}\right)
\end{aligned}
$$

The integrals $I_{3}, I_{4}$ are given by

$$
I_{3}=F_{1}^{\prime}(0)=\frac{\int_{0}^{\infty} \theta U(0, \theta) d \theta}{2 U(0,0)}=0.599, \quad I_{4}=\frac{2 F_{2}^{\prime}(0)}{U(0,0)}=\frac{\int_{0}^{\infty} \theta U^{2}(0, \theta) d \theta}{U^{2}(0,0)}=0.457,
$$

Equations (38) and (39) yield $\gamma_{0}=\frac{F_{0}}{\beta_{0}}\left(1+\frac{G_{0}^{2}}{F_{0}^{2}}\right) \frac{I_{4}}{I_{3}}$. For the magnetic field case,

$$
\frac{\beta_{0}}{r \alpha_{0}}=\frac{1}{3 m}
$$

Hence $\alpha \approx 3 m^{5 / 4} r^{-1 / 2}\left(\frac{I_{4}}{I_{3}}\right)^{1 / 2} \approx 2.62 m^{5 / 4} r^{-1 / 2}, \quad \beta \approx 0.78 m^{1 / 4} r^{1 / 2}$

$$
\gamma_{0} \approx 3 m^{5 / 4} r^{-1 / 2}\left(\frac{I_{4}}{I_{3}}\right)^{1 / 2} \approx 2.62 m^{5 / 4} r^{-1 / 2}, \phi=\tan \hat{\varepsilon}=\frac{\beta}{\alpha}=0.298 m^{-1} r
$$

The predictions (40) and (41) are tested against the full numerical solution given by Jasmine and Gajjar (2005) for large $m$ at a fixed Reynolds number. Fig. 2 shows that the results are consistent with equations (40) and (41).

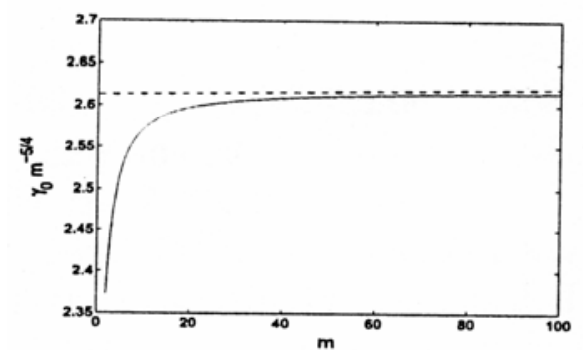

(a)

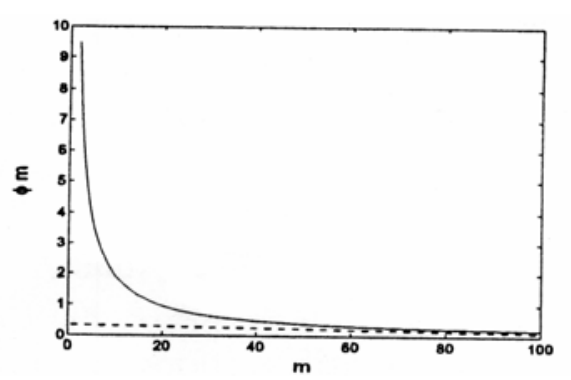

(b)

Fig. 2. Magnetic field $m$ versus (a) $\gamma_{0} m^{-5 / 4}$, and (b) $\phi m$ for fixed Reynolds number $R=1000$. The long curves show numerical results and doted lines show asymptotic ones. 


\section{CONCLUSIONS}

Asymptotic solutions for the upper branch neutral stability of the three dimensional disturbances imposed on Von-Ka'rma'n's boundary layer assuming large scale magnetic fields for non-zero frequency waves have been investigated. The asymptotic analysis of TG for non-zero frequency waves for the upper branch instability modes has been extended by imposing on the flow a magnetic field normal to the rotating disk. We have also extended investigations by Hall (1986) for stationary modes for the lower branch disturbances assuming large scale magnetic fields. A triple deck structure which governs the lower branch modes for a large scale magnetic field is displayed. Asympotic solution against the full numerical solution of the Navier-Stokes equations for large $m$ at a fixed Reynolds number has been tested. The behaviour of the wavenumbers and waveangle calculated from the eigenrelations is found to be consistent with the numerical results for large scale magnetic fields.

\section{REFERENCES}

Bassom, A. P. and J. S. B. Gajjar. 1988. Non-stationary crossflow vortices in a three dimensional boundary layer. Proc. Roy. Soc. London Ser. A, 417: 179-212.

Hall, P. 1986. An asymptotic investigation of the stationary modes of instability of the boundary layer on a rotating-disk. Proc. Roy. Soc. London Ser. A, 406: 93-106.

Jasmine, H. A. and J. S. B. Gajjar. 2005. Convective and absolute instability in the incompressible boundary layer on a rotating disk in the presence of a uniform magnetic field. J. Eng. Maths. 52: 337-353.

Mackherrell, S. O., 1987. A nonlinear asymptotic investigation of the stationary modes of instability of the three-dimensional boundary layer on a rotating disc. Proc. Roy. Soc. London Ser. A, 413: 497-513.

Malik, M. R., 1986. The neutral curve for stationary disturbances in rotating disk flow. J. Fluid Mech., 164: 275-287.

Smith, F. T., 1979. On the non-parallel flow stability of the Blasius bundary layer. Proc. Roy. Soc. London Ser. A, 366: 91-109.

Turkyilmazoglu, M. and J. S. B. Gajjar. 1998. Absolute and convective instability in the incompressible boundary layer on a rotating-disk. Report no. CLSCM, University of Manchester. 